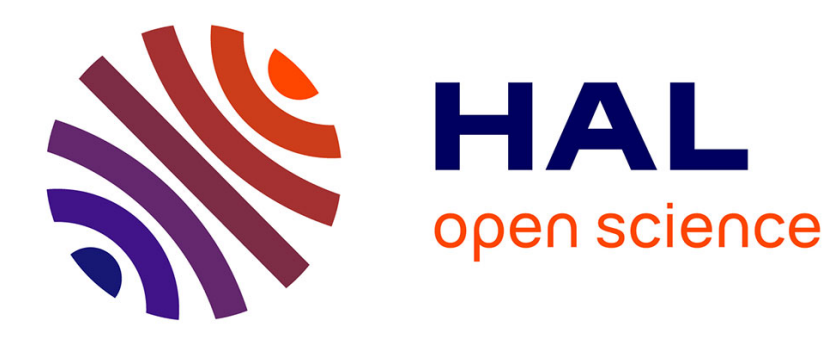

\title{
Description of heterogeneous dislocations distributions
}

\author{
G. Saada
}

\section{To cite this version:}

G. Saada. Description of heterogeneous dislocations distributions. Journal de Physique IV Proceedings, 1993, 03 (C7), pp.C7-585-C7-590. 10.1051/jp4:1993796 . jpa-00252216

\section{HAL Id: jpa-00252216 https://hal.science/jpa-00252216}

Submitted on 1 Jan 1993

HAL is a multi-disciplinary open access archive for the deposit and dissemination of scientific research documents, whether they are published or not. The documents may come from teaching and research institutions in France or abroad, or from public or private research centers.
L'archive ouverte pluridisciplinaire HAL, est destinée au dépôt et à la diffusion de documents scientifiques de niveau recherche, publiés ou non, émanant des établissements d'enseignement et de recherche français ou étrangers, des laboratoires publics ou privés. 


\title{
Description of heterogeneous dislocations distributions
}

\author{
G. SAADA
}

LEM CNRS ONERA, BP. 72, 92322 Chatillon cedex, France

\begin{abstract}
Different approximations for the description of the internal stress field due to dislocations walls are given and discussed.The influence of disorder and the edge effects due to incomplete walls are particularly discussed. Finally the relationship between the internal stress and the flow stress are discussed in these particular cases. It is shown that identifying the flow stress with the internal stress averaged in the volume of the crystal may be misleading. Consequences on the propagation of glide bands are also examined.
\end{abstract}

\section{1- Introduction}

During plastic deformation dislocations move, interact and organize themselves in a rather complex way. This results in the creation of internal stresses which influence the flow stress but should not be identified to the latter. The internal stress due to incompatible plastic strain,or equivalently to a given dislocation distribution, can be calculated by applying the methods developped by Kröner[1] and Eshelby[2],for example. However this is not sufficient to determine the flow stress, $i e$ the stress necessary to induce plastic deformation at a given strain rate. First it is well known that the average internal strain is zero in an homogeneous elastic medium. Second, due to the flexibility of dislocations, the flow stress saturates at a stress $\approx \mu \mathrm{b} / 1$, the so-called Orowan stress[3]. Here $\mu$ is the shear modulus, $\mathrm{b}$ the Burgers vector, 1 the average distance between the stress maxima.

Obviously, the treatment of the general problem is far from being solved, but one may obtain sensible results by describing relatively simple situations and compare them with the observed 
ones. However since all the results depend on the approximations,one has to analyze how robust the result is when the constraints arbitrarily imposed by the approximations are relaxed. In what follows, we shall analyze this situation for dislocation walls, since they are met very often in plastically deformed crystals, in fatigue, or during creep,or more simply at every planar surface limiting two regions where the plastic strain is different. In the next section, we shall analyze different kinds of dislocation walls, starting from infinite periodic planar dislocation walls,infinite disordered dislocation walls, finite dislocation walls. In section 3 , we shall apply the results to crystal plasticity. for simplicity sake, we shall restrict ourselves to elastically homogeneous solids.

\section{2- Dislocation walls[4].}

\section{2-1 Infinite continuous walls.}

Such walls exist at the limit between two regions where the plastic strain is homogeneous but exhibits a jump $\Delta \mathbf{E}$ (figure1a). In that case it is easily shown that the stress $\tau_{c}$ is homogeneous in the two half spaces limited by the wall and exhibits a jump $\Delta \tau_{c}$ at the wall. $\Delta \tau_{c}$ is a linear function of $\Delta \mathbf{E}$. It may eventually be 0 . Since the stress field does not decrease with the distance to the wall,such walls cannot exist alone,but may exist as a pair of opposite walls or dipolar walls(figure 1b).These results are valid in anisotropic elastic media.

Since crystal plasticity is discontinuous, continuous walls cannot exist. Therefore,we must analyze a slightly different situation, that of infinite, planar, periodic, dislocation walls.

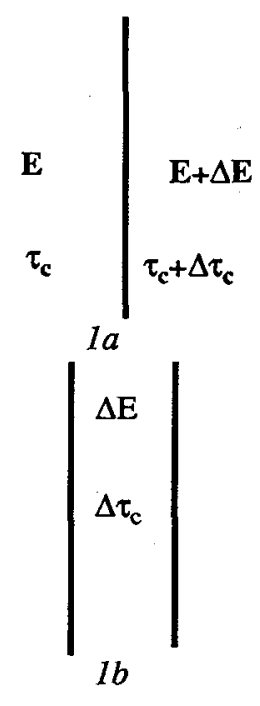

2-2 Infinite periodic planar dislocation walls.

The stress field $\tau_{\infty}$ of such a wall made of periodically distributed dislocations can be shown to consist in the sum of two contributions $\tau_{p}$ and $\tau_{\mathbf{L}} \cdot \tau_{\mathbf{p}}$ has the periodicity of the dislocation network and decreases as $\exp (-\mathrm{z} / \mathrm{l})$ with the distance $\mathrm{z}$ to the boundary, 1 being the period of the dislocations distribution. $\tau_{\mathrm{L}}$ is expressed as:

$$
\tau_{L}= \pm \tau_{0}
$$

Here the + sign corresponds to $z>0$, the - sign to $z<0, \tau_{0}$ is constant,eventually 0 , and depends only on the average dislocation distribution or on the difference in plastic strain on both side of the wall. Detailed formulae can be found in $[5,6,7]$. When $\tau_{0}$ is 0 ,one obtains the so-called 
low angle grain boundary $[8,9]$. Clearly, from formula 1 , whenever $\tau_{0} \neq 0$, walls must be associated in pairs cancelling their long range stress field which can be named dipolar

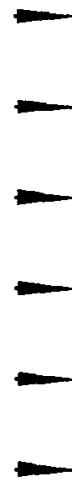

a

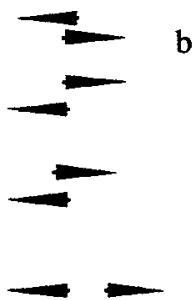

b
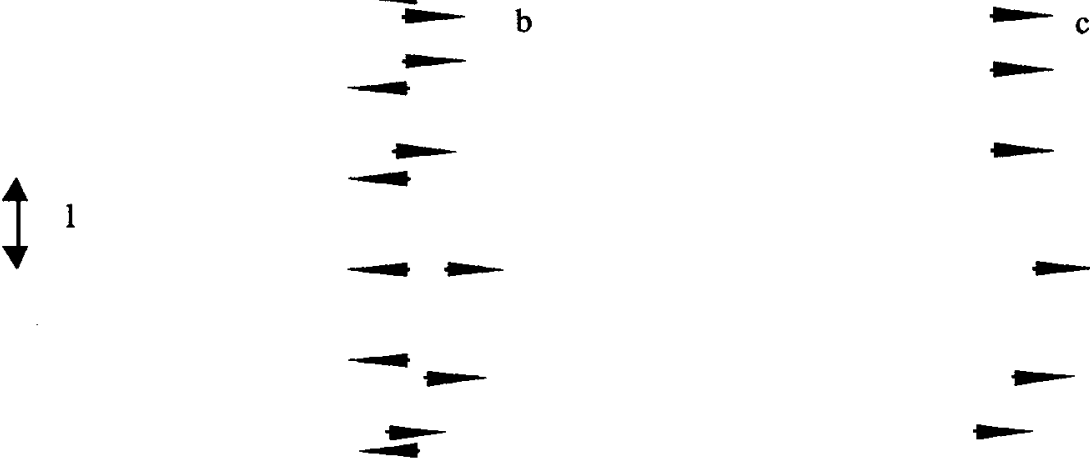

Figure 2

Periodic planar dislocation array(a),disordered dipole array(b)superposition of $a$ and $b$ is equivalent to a disordered array $(c)$

walls(Figure1). Mughrabi has described fatigue walls as dipolar walls[10].

It is therefore quite clear that replacing the approximation of continuous dislocation distribution, or of continuous strain, by a more general approximation of discontinuous periodic dislocation distribution, or plastic strain localized in periodically spaced planes, does not change the result at distance from the wall larger than 1 .Furthermore, $\tau_{0}$ scales with the average dislocation distribution, $i e$ as $1^{-1}$. On the other hand the internal stress $\tau_{p}$ has the period 1,therefore the corresponding Orowan stress scales as $1^{-1}$. Therefore the flow stress scales as $1^{-1}$, whatever the distance from the wall.

The above calculations are valid for both isotropic and anisotropic elasticity. They are also valid when there is more than one periodic dislocation array,and even in the case of a honeycomb network[7,11].

Let us now examine what happens when the dislocation distribution in the wall is disordered.

\section{2-3 Influence of disorder.}

We shall first consider a slightly disordered wall obtained by taking a periodic wall and giving the dislocations a random displacement $\delta$, of length $\delta$, such that $\delta / 1 \ll 1$.In that case, one may consider the elastic field of the disordered wall as the sum of the field of a periodic 
wall and the field of a disordered distribution of dislocation dipoles, $\tau_{d}[12]$. It can be shown that when the disorder is not correlated, any component of $\tau_{d}$,exhibits fluctuations which decrease slowly as $[4,12,13,14]$ :

$$
\tau_{\mathrm{d}} \approx \mu\left(<(\delta / 1)^{2}>\right)^{1 / 2}(\mathrm{~b} / 1)(1 / \mathrm{z})^{3 / 2}
$$

$\tau_{d}$ must be added to the field of the perfect wall. Formula (2) has a physical meaning at a distance $\mathrm{z}$ to the boundary larger than 1 . It is qualitatively valid both for isotropic and anisotropic elasticity. General formulae are given in[12] and show that the fluctuating stress can be appreciable in the case of epitaxial walls,or close to low angle boundaries, and in creep experiments as well as close to fatigue walls.

The case of large disorder has been treated in the particular case of a disordered array of parallel edge dislocations with Burgers vector perpendicular to the wall(disordered tilt boundary)[15]. The result is that the fluctuating stress field decreases as $(\mathrm{z} / \mathrm{h})^{-1 / 2}$. What is not clear in this result is the region of space where this stress is predominant. It is very likely that the result applies only at distance from the wall which are so large that it has no real physical significance.

\section{2-3 Edge effects.}

A general and convenient method has been given[4] to describe simply, the stress field of an incomplete planar periodic boundary. As shown in figure 3, we consider the slab as a part of an infinite periodic boundary. Then the stress field $\tau_{\mathrm{s}}$ of the incomplete boundary of width $w$ can be written as:

$$
\tau_{\mathrm{s}}=\tau_{\infty}-\tau_{\mathrm{c}}
$$

where $\tau_{\mathbf{c}}$ is the stress due to that part of the infinite boundary which is outside the slab.In order to calculate the stress inside the slab, it is a very good approximation to replace $\tau_{\mathbf{c}}$, by the stress field $\tau_{\mathbf{c}}{ }^{*}$ due to a continuous distribution of dislocations equal to the average value of $\tau_{\mathbf{c}}$. In the same manner, the stress outside the slab can be approximated by $\tau_{\mathrm{s}}{ }^{*}$, obtained by replacing the actual discrete distribution inside the slab by a continuous distribution.The result for a simple case is represented figure $4:$ at distance larger than 1 , the stress increases up to a value $\approx \mu \mathrm{b} / \mathrm{l}$, at a distance $\approx \mathrm{w}$ from the wall. Then it decreases roughly as $z^{-1}$. This applies in principle to both

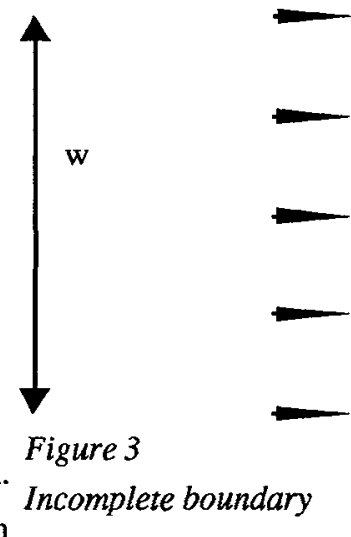


isotropic and anisotropic elasticity.

\section{3- Application to crystal plasticity}

Let us first consider the simple problem of evaluating the stress necessary to push a dislocation through a set of parallel dislocations walls. Obviously,if $\tau_{L}$ is zero, the volume fraction of the hard region is zero, and there is still an internal stress $\approx \mu \mathrm{b} / 1$ contributing to the flow stress. Even when $\tau_{\mathbf{L}} \neq 0$, it is easily verified, for example in the case of a hard zone close to the surface of a crystal undergoing plastic deformation, that

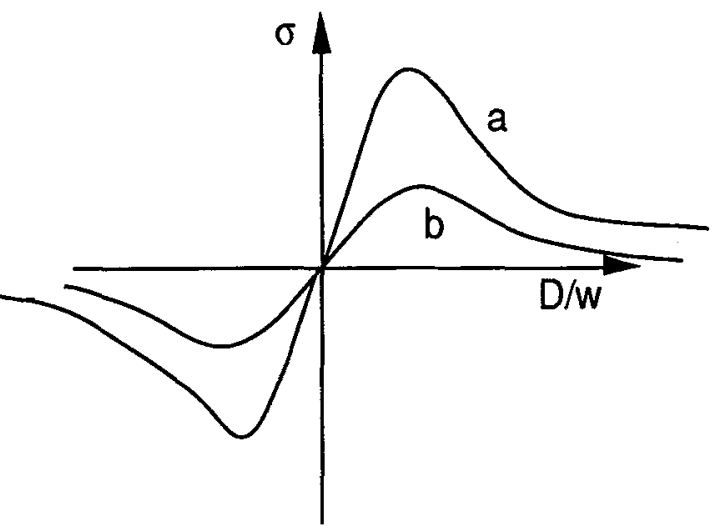

Figure4. Variation of the stress with the distance the flow stress cannot be calculated by $D$ to a wall of width $w$. taking a volume average of the internal b)close to the edge of the wall stress[16]. Therefore, even in the case of simple one dimensional problems, it is not correct to identify the flow stress with a volume average of the internal stress. Furthermore, the analysis we have made in the case of small disorder shows that the theoretical treatment of incompatible plastic strain at interfaces must take into account the slowly varying field defined by equation 2 .

If we consider now bounded walls, it is clear from figure 4 that the back stress on a glide band of width $w$ is larger at the center of the band and rather small at the edge of the band. This results in a lateral spreading of a glide band at an obstacle. It must be noted that,since the maximum of the forward stress is obtained at a distance of the order of $w$,from the glide band,the latter may proceed ahead after a sufficient widening. Therefore blocking a glide band results in the widening of the band. In turn, this widening produces a forward stress which induces forward propagation of glide at a distance w ahead of the obstacle.

\section{4-Conclusion}

It is rather easy to calculate the internal stress field of a dislocation wall,whether continuous, discrete, periodic, disordered,infinite, or finite. Departure from periodicity is shown to result in a stress field slowly decaying with distance. Edge effects due to finite wall result in establishing a correlation between the forward propagation and the lateral spreading of a glide 
band. Finally, in the case of obstacles like dislocation walls, it is not a valid assumption to compare the flow stress with an average internal stress.

Références

1- KRÖNER E. Physics of defects. North-Holland Amsterdam (1981) 215

2- ESHELBY J.D. Solid state physics 3. Academic press New-York (1956) 79

3-OROWAN E. Symposium on internal stress in metals and alloys. London, the Institute of Metals(1948) 451.

4-SAADA G. BOUCHAUD E. Acta metall.mater. 7 (1993) 2173

5-REY C. SAADA G. Phil.Mag. 33(1976) 825

6-SAADA G. Acta metall. 27 (1979) 921

7-SAADA G. Dislocation modelling of physical systems. Pergamon press New York(1980) 311

8-FRANK F.C. Report on the conf. on defects in crystalline solids. Physical society London (1955) 159

9-READ W.T. SHOCKLEY W. Imperfection in nearly perfects crystals Wiley New York (1952) 352

10-MUGHRABI H. Rev.Phys.Appl.23 (1988) 367

11-REY C. SAADA G. J.Phys. France 38 (1977) 721

12-BOUCHAUD E. SAADA G. Material Science Forum 126-128 (1993) 89

13-ZASIMCHUK E.E. SELITSER S.I. Sov.Phys.Solid State 26 (1984) 695

14-BOUCHAUD E. BOUCHAUD J.P. Phil.Mag.Lett.65 (1992) 339

15-NAZAROV A.A. ROMANOV A.E. VALIEV R.Z. Acta metll.mater.41 (1093) 1033

16-BONTEMPS C. VEYSSIERE P. SAADA G. Scripta Met.Mater.4 (1990) 2385 